\title{
Exploring Retraining-Free Speech Recognition for Intra-sentential Code-Switching
}

\author{
Zhen Huang, Xiaodan Zhuang, Daben Liu, Xiaoqiang Xiao, \\ Yuchen Zhang, Sabato Marco Siniscalchi \\ Apple Inc., 1 Broadway, Cambridge, MA 02142 \\ zhen_huangeapple, xiaodan@apple.com, daben_liudapple.com, xiaoqiang_xiaodapple.com, \\ yuchen_zhanglapple.com, ssiniscalchidapple.com
}

\begin{abstract}
In this paper, we present our initial efforts for building a codeswitching (CS) speech recognition system leveraging existing acoustic models (AMs) and language models (LMs), i.e., no training required, and specifically targeting intra-sentential switching. To achieve such an ambitious goal, new mechanisms for foreign pronunciation generation and language model (LM) enrichment have been devised. Specifically, we have designed an automatic approach to obtain high quality pronunciation of foreign language (FL) words in the native language (NL) phoneme set using existing acoustic phone decoders and an LSTM-based grapheme-to-phoneme (G2P) model. Improved accented pronunciations have thus been obtained by learning foreign pronunciations directly from data. Furthermore, a codeswitching LM was deployed by converting the original NL LM into a CS LM using translated word pairs and borrowing statistics for the NL LM. Experimental evidence clearly demonstrates that our approach better deals with accented foreign pronunciations than techniques based on human labeling. Moreover, our best system achieves a 55.5\% relative word error rate reduction from $34.4 \%$, obtained with a conventional monolingual ASR system, to $15.3 \%$ on an intra-sentential CS task without harming the monolingual recognition accuracy.

Index Terms: multilingual speech recognition, code-switching, grapheme-to-phoneme
\end{abstract}

\section{Introduction}

Code-switching (CS) is usually referred to as the situation where a speaker alternates between different languages within a single conversation, e.g., [1, 2, 3, 4]. Code-switching can be broadly divided into two groups [5]: inter-sentential switching - the alternation is between sentences (also called extrasentential), and intra-sentential switching - the alternation is within sentences (it can also include intra-word). With the rapidly growing of bilingual/multilingual population, CS is no longer a phenomenon relevant for minority languages, which are affected by majority languages, but it also concerns majority languages influenced by lingua francas, such as English and French, as properly pointed out in [6]. Despite the recent significant advances witnessed in the field of automatic speech recognition (ASR) [7], ASR systems have unfortunately still limited capability in tackling the code-switching problem, especially intra-sentential switching. Major challenges for CS speech recognition include different phone sets among languages, and insufficient intra-sentential code-switching training data.

In recent years, we have witnessed an increasing research effort to address the CS problem, and available approaches can mainly be divided into two groups: robust acoustic modeling, and language modeling. For example, a global phoneme set is constructed by merging phoneme sets from different languages, and a unified multilingual acoustic model (AM) is trained in [8, 9 10]. However, the goal of those studies on cross-language AMs is to develop ASR systems for new languages for which scarce or no training material is available. In the CS scenario, the native language (NL) AM should be used at the runtime, and the recognition accuracy and speed for the NL ASR should not be sacrificed. In [11], the authors compared four ASR approaches to tackle the CS task under the constraint that the NL $\mathrm{AM}$ is to be used at runtime. In those approaches, the lexicon in the foreign languages (FLs) are represented by the NL phoneme set through phone/senone mapping/merging using either knowledge-based or data-driven mapping techniques, and the NL acoustic model (AM) is employed to carry out recognition. It was shown that top recognition results were obtained by mapping each senone in the FL's AM to the senone in the NL's AM with the minimum Kullback-Leibler divergence. From a language model (LM) point of view, a statistical machine translation (SMT) based text generation technique was used in [12] to artificially augment the amount of training data to estimate more robust LMs. In [13], a multi-lingual LM is trained by combining monolingual LMs into a probabilistic finite state grammar. More recently, syntactic and semantic features were integrated into a factored language model to combat data scarcity in code-switching speech [14]. Recurrent neural networks (RNNs) and factored language models are instead combined in [15] for improved CS performance. In [16], the authors successfully used similar word pairs to borrow information from available (already trained) monolingual LMs and boost foreign low-frequency words. Since code-switching deals with more than a language, it is natural to exploite language identification, e.g., [17, 18, 19], or language diarization [6] to select among monolingual speech recognizers. Language identification was also employed in [12] to be combined with AM scores for better recognition performance. However, those approaches suffers from errors propagated by the language identification/diarization module to the ASR block.

In the afore-mentioned approaches, either ad-hoc acoustic and/or language models have to be built to specifically handle the CS task, or multiple language-dependent recognizers preceded by a language detection step have to be employed. In this paper, we demonstrate that leveraging existing monolingual ASR resources is possible to achieve effective intra-sentential code-switching without retraining any of the ASR models, namely the acoustic and language model, without sacrificing the recognition accuracy of the NL ASR system. We attain such a challenging goal by devising new mechanisms for foreign pronunciation generation, and LM enrichment. Specifically, we generate a high quality foreign pronunciation lexicon by obtaining the pronunciation of FL words in the NL phoneme set 
through acoustic phone decoding, and grapheme-to-phoneme conversion (G2P). Differently from [11], where phone/senone mapping and merging was the focus, we adopt data-driven techniques that learn foreign pronunciations directly from data, which in turn allows us to better deal with accented pronunciations. The experimental evidence interestingly demonstrates that data-driven acoustic phone decoding and G2P approaches perform favorably with respect to manual labeling. Moreover, we have obtained an enriched CS LM taking inspiration from [16]. Specifically, we have converted the initial NL LM into a CS LM by borrowing statistics/information from translated word pairs, which allows us to avoid LM retraining. Comparing to the state-of-the-art approach in [12], the proposed method doesn't need to construct a new phone set and retrain the AM, and also saves the efforts to retrain the LM. The retained LM by collecting/generating new CS data as in [12] can easily harm the recognition performance on NL. Experimental evidence on introducing FL words to a NL system with vocabulary size in the hundreds of thousands demonstrate that our best system is able to provide $55.5 \%$ relative word error rate reduction (from $34.4 \%$ to $15.3 \%$ ) on a code-switching testing set without harming the performance on general NL test sets.

\section{Foreign Pronunciation Generation}

$\mathrm{NL}$ refers the language whose phoneme set and AM are used in recognition. All other languages are indicated as FLs. For each FL word in a CS utterances, a native pronunciation representation in the NL's phoneme set is generated.

\subsection{Linguist Manual Labeling}

Manual labeling by experts can be the most straightforward way for this kind of pronunciation generation task. Linguists with knowledge of the NL and a FL can be hired to directly produce the lexicon of FL words with NL's phoneme set. The strength of this approach is pronunciations could be always reasonable for human perception; the shortcoming could be that linguists tend to label the FL words with the standard pronunciation, but native people always has some extent of accent when they speak foreign words in a CS scenario.

\subsection{Phone Decoding}

Phone decoding is a data-driven method to obtain pronunciations for the FL words. When the audio segments containing FL words, a NL ASR system can be used to decode those segments and thus obtain the possible phoneme sequences of the FL words. The audio segments mainly come from two sources: foreign people speaking FL words, and native people speaking FL words.

\subsubsection{Phoneme Confusion Network}

In harvesting audio segments for FL words, a particular word usually corresponds to more than one audio segment. The decoded phoneme sequences for those audio segments may vary a lot, and the process to obtain a good phoneme representation by summarizing all decoded phoneme sequences becomes crucial. In this paper, we adopt a ROVER-like [20] method based on phoneme confusion networks (CNs) to accomplish the summarization process. For example, let's suppose that there exist three (3) audio segments containing the English word "always," and the phone sequences generated with a Chinese ASR system are:
- OU W EI Z

- OUW I Z

- OU W EI S

Then the corresponding phoneme confusion network is displayed in Figure 1 where the numbers in parentheses represent the number of times a phoneme appears. From the phoneme confusion network in Figure 1, we can conclude that the best phoneme sequence (with most votes) for "always" is "OU W EI Z," and the second best could either be "OU W EI S," or "OU W IZ." For each FL words, we can retain an N-best representation.

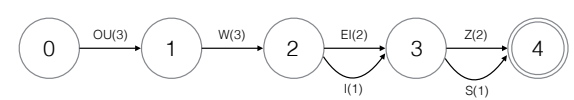

Figure 1: Phoneme confusion network for the word "always"

\subsubsection{Phone Decoding on $\boldsymbol{F L}$ words spoken by Foreign people (PDFF)}

In this paper, we suppose we have an already-trained monolingual speech recognition system for each language and there are plenty of corresponding training utterances. To harvest audio segments for foreign words, we use the FL speech recognition system to performance force alignment on its training utterances, by doing so, we can obtain the start/end time stamps of each word in those utterance. Then the audio segments containing concerned words are decoded with NL speech recognition system generating possible phoneme sequences for those words.

The advantage of this approach is that large amount of training data can be utilized to generate a lot of possible pronunciation representations and summaries them to get the best guesses, but a major concern is that those foreign words are spoken by foreign people, so to some extend, we are actually obtaining "standard" pronunciations while what we really care about is native people speaking foreign words with possible accents. To obtain such pronunciation guessing, we try to make use of the NL training data.

\subsubsection{Phone Decoding on FL words spoken by Native people (PDFN)}

Differently from FL training data, there are much fewer examples in NL training data that contain foreign words. Since we don't have native pronunciation in the native phoneme set for those words, it is not possible to obtain forced-alignments and find the corresponding audio segments (for those words) as shown in Section 2.2.2 However, foreign words appearing in the NL training data are the actual CS words, and although limited amount, these words can still be fundamental to find more relative pronunciation guesses for $\mathrm{CS}$ speech recognition. To obtain the audio segments of those foreign words in NL training datasets, we first utilize the PDFF system (see Section 2.2.2) to obtain an initial lexicon, and we then perform forced-alignment with such an initial lexicon to find the location of those words. After finding those audio segments, we can follow Section 2.2.2 to generate the pronunciation guesses.

\subsection{Grapheme-to-Phoneme Conversion}

Grapheme-to-Phoneme conversion(G2P) is the process of converting the written form of a word to its pronunciation. In this paper, we use a Long Short-Term Memory (LSTM) [21] based G2P model to translate the FL words from written form to pronunciation representation in NL's phoneme set. Our G2P model 
adopts an encoder-decoder architecture with attention mechanism [22] which is different from the LSTM G2P model proposed in [23]. There are two sources of data we used for the G2P model training:

(a) Standard NL lexicon containing linguist pronunciation labeling of both NL words and FL words. The amount of FL words is limited in this scenario;

(b) Phone decoding results on FL words spoken by native people (i.e., PDFN in Section 2.2.3

The training data (b) is added for the purpose of capturing the accents of NL speakers when they speak FL words. To obtain training data (b), we went through the following steps:

1 Collect audio recordings for the 5000 most popular FL words, which are spoken by NL speakers.

2 Use G2P trained with data (a) to generate 4 pronunciations from written form for each FL word used in Step1.

3 Use a NL phone decoder to generate additional 4 pronunciations for each FL word provided the audio collected in Step1.

4 The combined 8 pronunciations from Step2 and Step3 are rescored using the NL acoustic model, and the top 4 pronunciations with the highest acoustic model scores are retained for each FL word.

Then (a) and (b) are combined to form the final training data for the LSTM G2P model training.

\section{Language Model Enriching}

With the FL word pronunciation problem tackled, we still have to fix the LM part to deploy a robust CS system. LM training is a crucial problem in CS speech recognition, and the chief difficulty is in finding enough intra-sentential CS training data to build a reliable CS LM. In fact, the CS phenomenon does not happen as frequently in written form. However, it should not be difficult to convince ourselves that the lack of abundant CS text material is not a real concern, since the performance of the NL ASR system would be harmed if too much CS data is used to train the LM. Therefore, we started seeking into possible ways to overcome improve the LM in a CS system without collecting ad-hoc CS LM data, and we the idea proposed in [16 to be a sound solution to construct a CS LM. The idea is to borrow statistics/information in an already-trained LM, and we adopt and extend this idea in the present work.

In code-switching, we usually replaces a native word with the same meaning word (translated counterpart) in foreign language. For example, the monolingual Chinese utterance (meaning in English: "We play basketball")

$$
\text { - 我们打篮球 }
$$

can be spoken as

\section{- 我们打 basketball}

in code-switching scenarios. The code-switched words are a translated word pair “篮球-basketball”. Here "basketball” has the same meaning with “篮球”. In this example, when we construct the CS LM, we add the English (FL) word "basketball" into Chinese (NL) LM by copying the statistics/information of its translated counterpart “篮球 ”. By doing so, we borrow statistics/information of the word "篮球" in the NL LM for the added FL word "basketball". The effort of collecting/generating CS data and retraining LM can thereby be avoided using such an elegant statistics-borrowing method.

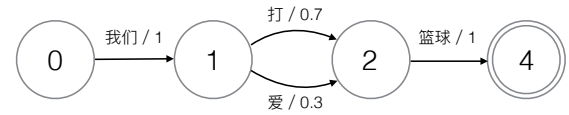

Figure 2: $N L L M G$

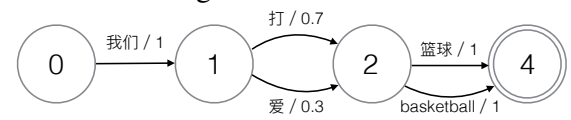

Figure 3: CS LM G' converted from NL LM G in Figure 2 by adding FL word "basketball"

Modern ASR systems usually use weighted finite-state transducers (WFST) [24] to present the LM as well as other components. We therefore show how to convert an NL LM into a CS LM in the context of WFST. In the LM WFST graph G, each transition/edge corresponds to a word, and the weight of the edge indicates the word's probability. Figure 2 is a toy example of a native LM's G graph. When we convert this toy NL LM into a CS LM concerning only the word "basketball" as follows: (i) Obtain the Chinese counterpart word "篮球” of "basketball," (ii) Find the edge in the WFST corresponding to the word “篮球," and (iii) Insert a parallel edge with the same weight into the WFST but carrying the the foreign word "basketbal." The above steps will result in the CS G graph shown in Figure 3

\section{Experimental Setup \& Results}

Our approach is validated using Chinese as the NL, and English as the FL.

\subsection{Experimental Setup}

Acoustic Model: The NL Chinese acoustic model is a FeedForward deep neural network (FFDNN) trained with filter bank features from 160 hours Chinese speech using cross-entropy and bMMI objective function. The FL English acoustic model utilize similar setup.

Lexicon: Table 1 is the lexicons we obtained by methods proposed in Section 2 The 1155 FL words are selected by their appearing frequencies in the English training data and also the probability occurring during Chinese-English code-switching. The $224 \mathrm{FL}$ words is a subset of the 1155 words which actually occur in the Chinese training data.

Code-Switching LM: Table 2 shows the code-switching LMs obtained following Section 3 We refer to the original Chinese LM as G0, the CS LM contains the 224 English word as G1, and the CS LM contains all the 1155 words as G2.

Testing sets: There are two test sets. The code-switching test set contains $107 \mathrm{CS}$ utterances. Each of the utterances contains 1 or more intra-sentential code-switchings. A typical one is like “我们打 basketball”. The FL words included in the CS test set is mainly a subset of the 224 English words afore-mentioend. The general test set contains 9677 utterances, most of which are pure Chinese utterances.

Evaluation Metric: Experimental results are reported in terms of a slightly modified version of the conventional word error rate (WER) metric, namely each Chinese character is treated as a word in the contest of CS speech recognition.

\subsection{Experimental Results}

Table 3 shows the CS performance of several systems having different lexicon and LM configurations. The CS system employing the original NL lexicon, namely L0, and the original 
Table 1: Lexicons used in the experimental evaluation

\begin{tabular}{|c||c|}
\hline \multicolumn{1}{|c|}{ Lexicons } & Configurations and Methods \\
\hline L0 & original NL lexicon (no FL words) \\
\hline L1 & 224 FL words by linguist labeling in Section 2.1 \\
\hline L2f & 1155 FL words by PDFF in Section 2.2.2 \\
\hline L2n & 224 FL words by PDFN in Section 2.2.3 \\
\hline L3a & 1155 FL words by G2P with data (a) in Section 2.3 \\
\hline L3ab & 1155 FL words by G2P with data (a) and (b) in Section 2.3 \\
\hline
\end{tabular}

Table 2: LMs with different FL vocabularies (see Section 3 )

\begin{tabular}{|c||c|}
\hline LMs & Configurations \\
\hline G0 & original NL LM (no FL words) \\
\hline G1 & CS LM with 224 FL words \\
\hline G2 & CS LM with 1155 FL words \\
\hline
\end{tabular}

LM, namely G0, is referred to as the baseline system. By comparing the second and third row in in Table 3 , we can easily argue that the proposed PDFF for generating the lexicon of FL words (see Section 2.2.2) along with enriched LM using 224 FL words (G1) delivers significant performance improvement over the baseline system. Indeed, the WER is reduced from $34.4 \%$ down to $24.3 \%$, which is equivalent to a word error rate reduction (WERR) of 29.4\%. The monolingual Chinese ASR system (baseline) cannot conversely properly recognize any foreign words, which are treated as out of vocabulary words. Moreover, we can observed that the proposed PDFF approach is equivalent, in terms of recognition performance, to the manual labeling of an expert (compare third and fourth rows). The latter might allow us to conclude that the data-driven method - without taking into account speaker's accents yet, is comparable to an expensive human-driven solution. By taking into account the speaker's accent using the L2n lexicon, we can further reduce the WER down to $20.7 \%$, which represents a WERR of $39.9 \%$, outperforming both human labeling, and the PDFN approach that tries to only generate "standard" pronunciations.

If an LSTM-based G2P approach trained on both the standard NL lexicon covering manually labeled NL and FL words (i.e., set (a) in Section 2.3, and the phone decoding results (i.e., set (b) in Section 2.3, a WER of $18.8 \%$ is attained, as displayed in the seventh row. By comparing the latter result with that shown in the sixth row in Table 3 which corresponds to G2P train on the set (a) only, we can see that taking speaker's accent into account has a beneficial effect on the recognition performance. Merging together all the pronunciation guesses from different sources brings the WER is down to $15.3 \%$, as shown in the eighth row in Table 3 , which accounts for a WERR of $55.5 \%$. The latter outcome seems to imply that those pronunciation generation methods are complementary to each other. Finally, we noticed that increasing the amount of FL words in the proposed CS LM does not provide any further improvement, but it does not harm the recognition performance on CS test set either. It is also important to verify that the addition of ad-hoc CS lexicons and LMs does not cause a drop in baseline ASR performance on pure Chinese utterances. To this end, we ran a new set of experiments on a more general data set (see Section 4.1). The corresponding results are reported the results in Table 4 and these results demonstrate that the performance degradation on a more general ASR task is ignorable.

Finally, the effect of tuning the weight-copying scale in LM enriching is given in Table 5 A scale factor is multiplied by the original NL edge's weight before being copied in the added parallel FL edge. A value greater than 1 implies a boost of the FL edge, whereas a value smaller than 1 suppresses the FL edge. From Table 5, we can see that boosting FL edges im-
Table 3: WERs (in \%) on CS test set by using different lexicon and LM configurations. Word error rates reductions (WERRs) are given in parentheses.

\begin{tabular}{|c||c||c|}
\hline 1 & Configuration & WER on CS \\
\hline 2 & L0+G0 (baseline) & $34.4 \%$ \\
\hline 4 & (L0+L2f)+G1 & $24.3 \%(29.4 \%)$ \\
\hline 3 & (L0+L1)+G1 & $24.3 \%(29.4 \%)$ \\
\hline 5 & (L0+L2n)+G1 & $20.7 \%(39.9 \%)$ \\
\hline 6 & (L0+L3a)+G1 & $21.6 \%(37.2 \%)$ \\
\hline 7 & (L0+L3ab)+G1 & $18.8 \%(45.3 \%)$ \\
\hline 8 & (L0+L1+L2f+L2n+L3ab)+G1 & $15.3 \%(55.5 \%)$ \\
\hline 9 & (L0+L1+L2f+L2n+L3ab)+G2 & $15.3 \%(55.5 \%)$ \\
\hline
\end{tabular}

Table 4: WERs (in \%) on the general test set.

\begin{tabular}{|c||c|}
\hline Configuration & WER on general \\
\hline L0+G0 (baseline) & $14.5 \%$ \\
\hline (L0+L1+L2f+L2n+L3ab)+G1 & $14.5 \%$ \\
\hline (L0+L1+L2f+L2n+L3ab)+G2 & $14.6 \%$ \\
\hline
\end{tabular}

proves the recognition performance on the CS test, but it also causes a degradation on the general test set. Suppressing FL edges won't harm the performance on the general set but causes a degradation on the CS set. That can easily be understood by bearing in mind that errors on the CS test set are mainly caused by unrecognized FL words; therefore, better results can be obtained by boosting the FL edges. However, there are nearly no FL words in a more general test set, and that explains the drop in performance.

Table 5: Effects on tuning the weight-copying scale with configuration of $(L O+L 1+L 2 f+L 2 n+L 3 a b)+G 2$.

\begin{tabular}{|c||c|c|}
\hline Scale & WER on general & WER on CS \\
\hline 1 & $14.5 \%$ & $15.3 \%$ \\
\hline 1.5 & $14.9 \%$ & $12.8 \%$ \\
\hline 0.667 & $14.5 \%$ & $18.6 \%$ \\
\hline
\end{tabular}

\section{Conclusion}

In this work, we build a intra-sentential code-switching ASR prototype by leveraging existing monolingual systems without either acoustic, or language model retraining. Our approach focuses on data-driven pronunciation generation for FL words and takes into account speakers' accent. Moreover, we also devise a simply CS LM generation approach by enriching existing monolingual LM leveraging word pairs. The significant accuracy improvement on CS test data together with retention of LM accuracy on a more general speech task highlights the effectiveness of our idea. A major limitation of the proposed approach concerns the LM enriching method, which may not handle the word reordering problem. To clear ideas, let's consider the English word "tomorrow" that corresponds to the Chinese word “明天”. In “I will go to school tomorrow" the adverb “tomorrow" appears at the end of the sentence, but the same sentence in Chinese, namely “我明天去学校“" would places “明天” in the middle of the sentence. In a CS sentence having "tomorrow" as the FL word, "tomorrow" would rarely appear in the middle of the sentence, as it should in Chinese, and that may make the use of the statistics borrowed from the NL LM for the CS LM not accurate. We will try to tackle this issue by combing the proposed statistic-borrowing and the traditional data augmentation methods. 


\section{References}

[1] S. Poplack, "Sometimes ill start a sentence in spanish y termino en español: toward a typology of code-switching1," Linguistics, vol. 18, pp. 581-618, 1980.

[2] D. Sankoff and S. Poplack, "A formal grammar for codeswitching," Research on Language \& Social Interaction, vol. 14, pp. 3-45, 1981 .

[3] L. Milroy and P. Muysken, One speaker, two languages: Cross disciplinary perspectives on code-switching. Cambridge University Press, 1995.

[4] P. Auer, Code-switching in conversation: Language, interaction and identity. Routledge, 2013.

[5] C. Myers-Scotton, A formal grammar for code-switching. Oxford: Clarendon Press, 1993.

[6] E. Yilmaz, M. McLaren, H. V. den Heuvel, and D. A. V. Leeuwen, "Language diarization fro semi-supervised bilingual acoustic model training," in Proc. ASRU. ISCA, 2017, pp. 91-96.

[7] G. Hinton, L. Deng, D. Yu, G. Dahl, A. r. Mohamed, N. Jaitly, A. Senior, V. Vanhoucke, P. Nguyen, T. Sainath, and B. Kingsbury, "Deep neural networks for acoustic modeling in speech recognition," Signal Processing Magazine, 2012.

[8] S. Yu, S. Zhang, and B. Xu, "Chinese-english bilingual phone modeling for cross-language speech recognition," in Proc. ICASSP, vol. 1. IEEE, 2004, pp. I-917.

[9] C.-L. Huang and C.-H. Wu, "Generation of phonetic units for mixed-language speech recognition based on acoustic and contextual analysis," IEEE Trans. Computers, vol. 56, no. 9, pp. 12251233, 2007

[10] H. Lin, L. Deng, D. Yu, Y. Gong, A. Acero, and C.-H. Lee, "A study on multilingual acoustic modeling for large vocabulary asr," in Proc. ICASSP. IEEE, 2009, pp. 4333-4336.

[11] D. Yu, L. Deng, P. Liu, J. Wu, Y. Gong, and A. Acero, "Crosslingual speech recognition under runtime resource constraints," in Proc. ICASSP. IEEE, 2009, pp. 4193-4196.

[12] N. T. Vu, D.-C. Lyu, J. Weiner, D. Telaar, T. Schlippe, F. Blaicher, E.-S. Chng, T. Schultz, and H. Li, "A first speech recognition system for mandarin-english code-switch conversational speech," in Proc. ICASSP. IEEE, 2012, pp. 4889-4892.

[13] F. Weng, H. Bratt, L. Neumeyer, and A. Stolcke, "A study of multilingual speech recognition." in Proc. EUROSPEECH, vol. 1997, 1997, pp. 359-362.

[14] H. H. Adel, N. T. Vu, K. Kirchhoff, D. Telaar, and T. Schultz, "Syntactic and semantic features for code-switching factored language models," IEEE Trans. Audio, Speech, and Language Processing, vol. 23, no. 3, pp. 431-440, 2015.

[15] H. Adel, D. Telaar, N. T. Vu, K. Kirchhoff, and T. Schultz, "Combining recurrent neural networks and factored language models during decoding of code-switching speech." in Proc. INTERSPEECH, 2014, pp. 1415-1419.

[16] X. Ma, X. Wang, D. Wang, and Z. Zhang, "Recognize foreign low-frequency words with similar pairs," in Proc. INTERSPEECH, vol. 2015.

[17] A. Waibel, H. Soltau, T. Schultz, T. Schaaf, and F. Metze, "Multilingual speech recognition," in Verbmobil: Foundations of Speech-to-Speech Translation. Springer, 2000, pp. 33-45.

[18] H. Lin, J.-T. Huang, F. Beaufays, B. Strope, and Y.-H. Sung, "Recognition of multilingual speech in mobile applications," in Proc. ICASSP. IEEE, 2012, pp. 4881-4884.

[19] J. Gonzalez-Dominguez, D. Eustis, I. Lopez-Moreno, A. Senior, F. Beaufays, and P. J. Moreno, "A real-time end-to-end multilingual speech recognition architecture," IEEE Journal of Selected Topics in Signal Processing, vol. 9, no. 4, pp. 749-759, 2015.

[20] J. G. Fiscus, "A post-processing system to yield reduced word error rates: Recognizer output voting error reduction (rover)," in Proc. ASRU. IEEE, 1997, pp. 347-354.
[21] S. Hochreiter and J. Schmidhuber, "Long short-term memory," Neural computation, vol. 9, no. 8, pp. 1735-1780, 1997.

[22] D. Bahdanau, K. Cho, and Y. Bengio, "Neural machine translation by jointly learning to align and translate," 2015.

[23] K. Rao, F. Peng, H. Sak, and F. Beaufays, "Grapheme-to-phoneme conversion using long short-term memory recurrent neural networks," in Proc. ICASSP. IEEE, 2015, pp. 4225-4229.

[24] M. Mohri, F. Pereira, and M. Riley, "Weighted finite-state transducers in speech recognition," Computer Speech \& Language, vol. 16, no. 1, pp. 69-88, 2002. 